\title{
Identification of a long non-coding RNA regulator of liver carcinoma cell survival
}

\author{
Yulia Rybakova (1) 1,2, John T. Gonzalez(10), Roman Bogorad (10', Vikash P. Chauhan', Yize L. Dong, \\ Charles A. Whittaker ${ }^{1}$, Timofei Zatsepin (1) ${ }^{2}$, Victor Koteliansky ${ }^{2}$ and Daniel G. Anderson (1) ${ }^{1,5,6,7}$
}

\begin{abstract}
Genomic studies have significantly improved our understanding of hepatocellular carcinoma (HCC) biology and have led to the discovery of multiple protein-coding genes driving hepatocarcinogenesis. In addition, these studies have identified thousands of new non-coding transcripts deregulated in HCC. We hypothesize that some of these transcripts may be involved in disease progression. Long non-coding RNAs are a large class of non-coding transcripts which participate in the regulation of virtually all cellular functions. However, a majority of IncRNAs remain dramatically understudied. Here, we applied a pooled shRNA-based screen to identify IncRNAs essential for HCC cell survival. We validated our screening results using RNAi, CRISPRi, and antisense oligonucleotides. We found a IncRNA, termed ASTILCS, that is critical for HCC cell growth and is overexpressed in tumors from HCC patients. We demonstrated that HCC cell death upon ASTILCS knockdown is associated with apoptosis induction and downregulation of a neighboring gene, protein tyrosine kinase 2 (PTK2), a crucial protein for HCC cell survival. Taken together, our study describes a new, non-coding RNA regulator of HCC.
\end{abstract}

\section{Introduction}

Liver cancer is one of the leading causes of cancer mortality worldwide, accounting for more than 700,000 deaths per year ${ }^{1}$. Hepatocellular carcinoma (HCC) is the most frequent subtype of liver cancer. Despite recent progress in HCC treatment it remains one of the deadliest types of cancer ${ }^{1,2}$. Notably, the incidence of $\mathrm{HCC}$ has been increasing in recent decades, making $\mathrm{HCC}$ one of the fastest-growing causes of death worldwide ${ }^{3}$. This poor prognosis underlines the need for new effective therapies. Better understanding of the molecular mechanisms regulating HCC progression may yield new potential drug targets.

A meta-analysis of human HCC datasets revealed 935 genes for which RNA expression was significantly

\footnotetext{
Correspondence: Daniel G. Anderson (dgander@mit.edu)

'David H. Koch Institute for Integrative Cancer Research, Massachusetts Institute of Technology, Cambridge, MA 02142, USA

${ }^{2}$ Skolkovo Institute of Science and Technology, Moscow 121205, Russia

Full list of author information is available at the end of the article

Edited by B. Rotblat
}

dysregulated in HCC samples compared to healthy tissues $^{4}$. Further Gene Ontology analysis of these genes identified several gene networks associated with HCC progression. Among them were upregulation of cell proliferation, downregulation of apoptosis, loss of hepatocyte differentiation, immunosuppression, and activation of proteins acting at an epigenetic level ${ }^{4}$. Comprehensive genomic profiling of patient HCC samples and their comparison with healthy tissues have helped uncover molecular changes promoting the above phenotypic features of $\mathrm{HCC}^{5-9}$. Among them, mutations leading to activation of the WNT signaling pathway were most common $^{5,6}$, implicating the WNT pathway as a major driver of hepatocarcinogenesis ${ }^{7}$. Moreover, activation of the WNT pathway is associated with an immunosuppressive microenvironment, another hallmark of HCC progression $^{8,9}$, which emphasizes the role of WNT pathway activity in HCC progression. Other common mutations affected the TERT promoter, TP53, genes regulating cell cycle, PI3K-AKT-mTOR signaling and cell differentiation $^{5,6}$. Notably, up to $50 \%$ of clinical HCC samples

\section{(c) The Author(s) 2021}

(c) (i) Open Access This article is licensed under a Creative Commons Attribution 4.0 International License, which permits use, sharing, adaptation, distribution and reproduction cc) in any medium or format, as long as you give appropriate credit to the original author(s) and the source, provide a link to the Creative Commons license, and indicate if changes were made. The images or other third party material in this article are included in the article's Creative Commons license, unless indicated otherwise in a credit line to the material. If material is not included in the article's Creative Commons license and your intended use is not permitted by statutory regulation or exceeds the permitted use, you will need to obtain permission directly from the copyright holder. To view a copy of this license, visit http://creativecommons.org/licenses/by/4.0/. 
reported in different studies have a mutation in chromatin modifiers ${ }^{5,6}$, indicating the importance of epigenetic regulation in HCC development.

Besides shedding light on the roles of protein-coding genes, integrative genomic studies have revealed that the majority $(>70 \%)$ of transcribed sequences in the human genome participate in cell function regulation without producing a protein ${ }^{10}$. Long non-coding RNAs (lncRNAs) are defined as non-coding transcripts longer than 200 nucleotides and represent a large class of non-coding elements, comprising more than 50,000 annotated transcripts to date ${ }^{11,12}$. Pertinently, hundreds of lncRNAs are recurrently deregulated in HCC, suggesting potential roles in hepatocarcinogenesis. Co-expression network analysis determined that these IncRNAs were associated with cell proliferation, metastasis, immune response, and liver metabolism-hallmarks of HCC progression ${ }^{13,14}$. While the pathogenic roles of some of these lncRNAs (e.g. HULC, H19, HOTAIR, HOTTIP, DANCR) have already been described ${ }^{15}$, a plurality of lncRNA transcripts remain largely uncharacterized. Discovery of novel lncRNAs and their intracellular functions promises to expand our knowledge of HCC cellular physiology and may provide the basis for new therapeutic modalities.

Currently, lncRNA functions can hardly be predicted based on their sequence. Instead, subcellular localization, transcript abundance, and functional genomic screens can help to efficiently narrow down possible lncRNA biological roles and molecular functions ${ }^{16-19}$. For instance, IncRNAs located mainly in the nucleus typically function as transcription regulators of local genes (in cis) or distant genes (in trans) ${ }^{18}$. Cytoplasmic lncRNAs are more likely to regulate protein production, formation of posttranslational modifications, and sequestration of miRNAs or RNA-binding proteins ${ }^{19}$. Transcript abundance can provide another hint about lncRNA function. For example, low-abundance transcripts tend to function in cis because their low concentration makes diffusion a barrier to activity at long distances from the transcription site. Abundant lncRNAs, on the other hand, can achieve high concentrations at multiple target regions, including those outside of the nucleus and therefore often function in $\operatorname{trans}^{16,17}$. Finally, pooled functional genetic screens are a powerful tool allowing for parallel perturbation of multiple genes to select for those that are critical for a phenotype or function ${ }^{20,21}$. Recently, genome-wide screens have made it possible to identify lncRNAs involved in a wide variety of cellular functions including cell proliferation, drug resistance, autophagy, tissue homeostasis, and cell differentiation ${ }^{22-26}$.

RNA interference (RNAi) is an effective method for transient silencing of gene expression and therefore is an instrument for loss-of-function genetic screens ${ }^{20,27}$. Previously, it was reported that RNAi-mediated gene silencing is restricted to the cytoplasm, limiting targeting of nuclear transcripts. However, recent studies suggest RNAi presence and activity in the mammalian nucleus as well, although with less efficiency ${ }^{28-30}$. Clustered regularly interspaced short palindromic repeat interference (CRIS$\mathrm{PRi}$ ) is another potent technique for lncRNA silen$\operatorname{cing}^{21,23,25}$. Given that majority of IncRNA promoters is poorly annotated and IncRNAs often overlap with protein-coding genes (or their promoters/enhancers), application of CRISPRi to regulate a lncRNA overlapping with other transcripts might distort expression of those transcripts, confounding data interpretation ${ }^{31}$. Thus, in our screen we have chosen to perturb IncRNA at the transcript level. We performed an shRNA-based pooled screen to identify lncRNAs essential for the survival of the human HCC cell line HUH7. Based on the lncRNA expression profile of these cells, we designed a lentiviral shRNA library targeting all identified lncRNAs. Using this library, we performed a loss-of-function genetic screen and found that lncRNA ENST00000501440.1 is critical for HUH7 cell growth. We named this lncRNA ASTILCS (AntiSense Transcript Important for Liver Carcinoma Survival). Importantly, in patient data, ASTILCS is significantly overexpressed in HCC compared to normal tissues. Further, using gene expression manipulation techniques, we demonstrate that ASTILCS knockdown results in apoptosis induction and HCC cell death. Finally, we show that ASTILCS knockdown correlates with downregulation of a neighboring gene expressing Protein Tyrosine Kinase 2 (PTK2), the silencing of which results in HCC cell death.

\section{Materials and methods Cell culture}

Human HCC HUH7 cell line was a gift from Prof. Jay Horton (UT Southwestern Medical Center). HUH7 and HEK293ft cell lines were grown in Dulbecco's modified Eagle's medium with L-glutamine (DMEM, Gibco ${ }^{\mathrm{TM}}$ ) supplemented with $4.5 \mathrm{mg} / \mathrm{ml}$ glucose, $50 \mu \mathrm{g} / \mathrm{ml}$ gentamicin sulfate (Sigma), $25 \mathrm{mM}$ HEPES $\left(\mathrm{Gibco}^{\mathrm{TM}}\right)$, and $10 \%$ heatinactivated fetal bovine serum (FBS, Gibco ${ }^{\mathrm{TM}}$ ). All cells were cultured at $37^{\circ} \mathrm{C}, 5 \% \mathrm{CO}_{2}$. When the cells reached a $70-80 \%$ monolayer, they were detached from the flask using $0.25 \%$ Trypsin-EDTA $\left(\right.$ Gibco $^{\mathrm{TM}}$ ) solution and split 1:10. Concentrations for selection agents were determined using killing curve: $2.5 \mu \mathrm{g} / \mathrm{ml}$ puromycin (Sigma), $0.75 \mathrm{mg} / \mathrm{ml} \mathrm{G}-480$ (Sigma). The cells were tested for mycoplasma contamination.

\section{RNA sequencing and data analysis}

Samples were prepared using strand-specific Ribo-Zero kit and RNA sequencing was performed by MIT BioMicro Center (https://openwetware.org/wiki/BioMicroCenter: Software\#BMC-BCC_Pipeline). Reads were aligned to 
transcripts derived from the hg19 assembly and the Ensembl version 68 non-coding RNA annotation (noncoding genes) or the full Ensembl 68 annotation (proteincoding genes) using Bowtie version $1.01^{32}$ and gene expression was summarized using RSEM version 1.2.3 $3^{33}$.

\section{Genome-wide screening}

Based on HUH7 RNA-sequencing results (Supplemental Fig. 1, Supplemental Table 1), we designed a library of 7873 shRNA vectors allowing to do knockdown of the identified 1618 lncRNAs based on $\mathrm{RNAi}^{34,35}$. The library was developed, synthesized, and packed into lentivirus by the RNAi Consortium at the Broad Institute ${ }^{36}$. The shRNA sequences were assembled into a pLKO.1 lentiviral backbone, containing a puromycin resistance marker to allow for the antibiotic selection of transduced cells. CMV-VSV-G and psPAX2 plasmids were used for lentiviral packaging. The lentiviral library contained four to five shRNAs per target lncRNA and was applied at a low multiplicity of infection (MOI) equal to 0.3 . Two days after lentiviral library exposure, infected cells were selected for 4 days on puromycin. To assess effects of shRNAs on cell survival, the selected cells were cultured for 4 more weeks maintaining an shRNA representation of 500 (i.e. each shRNA was expressed on average by 500 cells). The input pooled shRNA plasmid library before virus production was also sequenced and used as a control.

\section{Next generation sequencing}

Samples for Illumina sequencing were prepared following "One Step PCR Preparation of Samples for Illumina Sequencing" protocol from The RNAi Consortium (https://portals.broadinstitute.org/gpp/public/resources/ protocols). Briefly, gDNA was isolated using the QIAamp DNA Blood Maxi Kit (Qiagen). Illumina adapter sequences with five-letter barcodes were used to PCR amplify the shRNA-expressing cassette. The samples were multiplexed and sequenced by MIT BioMicroCenter using HiSeq2000 platform. The samples were processed using the $\mathrm{BMC} / \mathrm{BCC}$ 1.5.2 pipeline (updated on 08/12/ 2016). Adapter sequence GGAAAGGACGAGGTACC was trimmed from reads using Cutadapt version 1.4.2 $2^{37}$. Trimmed reads were then aligned target consisting of the 7873 sequence shRNA library with BWA version $0.7 \cdot 10^{38}$. Mapped reads were summarized and parsed using SAMtools version $1.3^{39}$ and custom Perl scripts. The resulting count table was tested for differential representation using DESeq2 version $1.10 .1^{40}$ running under $\mathrm{R}$ version 3.2.3. Differential expression data was visualized using Tibco Spotfire Analyst version 7.11.1.

\section{Molecular cloning}

shRNAs from the library (Supplemental Table 2) were annealed and cloned into a pLKO.1_neo plasmid (a gift from Prof. Sheila Stewart; Addgene plasmid \# 13425; http://n2t.net/addgene:13425; RRID:Addgene_13425) using a protocol from Wiederschain et $\mathrm{al}^{41}$. Two shRNAs designed to target mCherry were used as controls. Briefly, oligos were resuspended in water to a final concentration of $100 \mu \mathrm{M} .11 .25 \mu \mathrm{l}$ of each oligo (top and bottom) were mixed with $2.5 \mu \mathrm{l}$ of $10 \times$ annealing buffer $(1 \mathrm{M} \mathrm{NaCl}$ (Sigma), $100 \mathrm{mM}$ Tris-HCl (Sigma), $\mathrm{pH}=$ 7.4) and annealed at $95^{\circ} \mathrm{C}$ using a water bath. The pLKO.1_neo plasmid was digested using AgeI and EcoRI restriction enzymes and purified on 1\% agarose gel. Next, oligo mixture was diluted 1:400 in $0.5 \times$ annealing buffer and ligated with the digested PLKO.1_neo plasmid using T4 DNA ligase (NEB) ( $3 \mathrm{~h}$ at RT). $2 \mu$ lof the ligation mixture was used to transform $10 \mu \mathrm{l}$ of One Shot competent Stbl3 E. coli cells (Invitrogen) according to manufacturers' instructions. Transformed bacteria were plated on LB-agar plates (Teknova) with $100 \mu \mathrm{g} /$ $\mathrm{mL}$ ampicillin (Sigma) and incubated overnight. Individual colonies were picked, inoculated in $3 \mathrm{ml}$ of LB (Sigma) with ampicillin to start miniprep cultures and incubated for $24 \mathrm{~h}$. Miniprep DNA was isolated using QIAGEN Plasmid Mini Kit (Qiagen). shRNA sequences were confirmed by Sanger sequencing (performed by Quintara Biosciences).

sgRNAs (Supplemental Table 3) were designed using the Broad Institute's GPP sgRNA Designer ${ }^{42,43}$. Two sgRNAs targeting mouse XIST and blasted against human genome and transcriptome to avoid off-targets were used as controls. Then, the sgRNAs were assembled into a plasmid expressing dead Cas9 (dCas9, Cas9 without endonuclease activity) fused with a transcription inhibitor, the Krüppel-associated box (KRAB) transcriptional repression domain, in a lentiviral backbone containing a puromycin resistance sequence (pLV hU6-sgRNA hUbC-dCas9-KRAB-T2a-Puro, a gift from Prof. Charles Gersbach, Addgene plasmid \# 71236; http://n2t.net/ addgene:71236; RRID:Addgene_71236 ${ }^{44}$ using Golden Gate assembly reaction as described in ref. ${ }^{45} .2 \mu \mathrm{l}$ of the ligation mixture were used to transform $10 \mu \mathrm{l}$ of NEB stable competent $E$. coli (NEB) according to manufacturers' instructions. Transformed bacteria were plated on LB-agar plates with $100 \mu \mathrm{g} / \mathrm{mL}$ ampicillin and incubated overnight. Individual colonies were picked, inoculated in $3 \mathrm{ml}$ of LB (Sigma) with ampicillin to start miniprep cultures and incubated for $24 \mathrm{~h}$. Miniprep DNA was isolated using QIAGEN Plasmid Mini Kit (Qiagen). sgRNA sequences were confirmed by Sanger sequencing (performed by Quintara Biosciences).

To create a plasmid expressing ASTILCS, it's full sequence was used to substitute GFP in TRC209 lentiviral plasmid (PGK-Hygro-EF1a-GFP, gift from the Broad $\mathrm{GPP})^{23}$. The cloning and sequence validation were done by Genscript Biotech. 


\section{Lentivirus production and transduction}

For transduction, plasmids were packaged into lentivirus through transfection of the plasmids with a packaging plasmid (psPAX2 was a gift from Prof. Didier Trono (Addgene plasmid \# 12260; http://n2t.net/addgene:12260; RRID:Addgene_12260) and an envelope plasmid CMVVSV-G was a gift from Prof. Weinberg (Addgene plasmid \# 8454; http://n2t.net/addgene:8454; RRID:Addgene_8454) ${ }^{46}$ using TransIT-LT1 Transfection Reagent (Mirus Bio). $\sim 300,000$ HEK293ft cells were plated per well into a sx-well plate and incubated overnight. $0.4 \mu \mathrm{g}$ PAX2, $0.15 \mathrm{VSV}-\mathrm{G}$ and $3.3 \mu \mathrm{g}$ plasmid of interest were added to $600 \mu \mathrm{l} \mathrm{Opti-}$ MEM $\left(\mathrm{Gibco}^{\mathrm{TM}}\right)$ and mixed with an equal volume of OptiMEM containing $4 \mu \mathrm{l}$ of TransIT-LT1. The mixture was incubated at RT for $20 \mathrm{~min}$ and transferred to the well. The volume was brought to $600 \mathrm{ml}$ per well with the culture media and incubated overnight. On the following day, the media was changed. Media with lentiviral particles was collected after $48 \mathrm{~h}$ and snap-frozen in liquid nitrogen. All shRNA/sgRNA plasmids were produced in parallel.

\section{Arrayed screening}

Equal numbers of HUH7 cells ( 5000) were plated in a 96-well plate and transduced with $5 \mu \mathrm{l}$ of shRNAs or $2 \mu \mathrm{l}$ of sgRNAs packed into lentiviral particles, so that each well received only one type of shRNA/sgRNA. A plasmid expressing green fluorescent protein (GFP) (pLJM1-EGFP was a gift from Prof. David Sabatini (Addgene plasmid \# 19319; http://n2t.net/addgene:19319; RRID:Addgene 19319) $)^{47}$, but not caring antibiotic resistance marker was also packed into lentiviral particles and used as a positive control for transduction and antibiotic selection. After an overnight incubation the cell media was changed. Two days after the lentiviral transduction, a selection reagent (G-480 or puromycin, respectively) was added to the culture media to select for cells containing the shRNA/ sgRNA expressing plasmids. Once the selection was completed (i.e. all non-infected GFP-treated cells were dead), cell survival was measured using Cell Titer assay.

\section{Cell survival assay}

HUH7 cell survival was analyzed using the CellTiter-Glo ${ }^{\circledR}$ Luminescent Cell Viability Assay (Promega) according to the manufacturer's protocol. Luminescence was measured with the microplate reader Tecan Infinite ${ }^{\circledR} 200$ PRO.

\section{Cell proliferation assay}

Cells were plated at low density in 96-well plates (2000 cells/well). Cell number analysis using cell titer assay was performed at 1, 2, 3, and 5 days afterwards. For growth curves analysis, doubling time was calculated from the exponential portion of the cell growth curve using the following equation: $T_{\mathrm{d}}=0.693 \mathrm{t} / \ln \left(N_{t} / N_{0}\right)$, where $t$ is the time (in days), $N_{0}$ is initial cell number, $N_{\mathrm{t}}$ is cell number on day $t$.

\section{Gene expression analysis}

For single tube reactions (Fig. 3G) RNA was isolated using Omega Bio-tek's E.Z.N.A. ${ }^{\circledR}$ Total RNA Kit I isolation kit according to manufacturers' instructions. Separation and purification of cytoplasmic and nuclear RNA (Fig. 3E) were done using Cytoplasmic and Nuclear RNA Purification Kit (Norgen Biotek Corp.) also following manufacturers' instructions. Reverse transcription reaction was performed using Applied Biosystems ${ }^{\mathrm{TM}}$ HighCapacity RNA-to-cDNA ${ }^{\mathrm{TM}}$ Kit and $1 \mu \mathrm{g}$ of RNA. RNA levels were assessed by qPCR using Power SYBR ${ }^{\mathrm{TM}}$ Green PCR Master Mix (Applied Biosystems ${ }^{\mathrm{TM}}$ ). For highthroughput experiments (all other RNA expression figures) RNA isolation, reverse transcription reaction, and qPCR was performed using Power SYBR ${ }^{\mathrm{TM}}$ Green Cells-to$\mathrm{CT}^{\mathrm{TM}}$ Kit (Ambion) according to manufacturers' instructions. TaqMan Fast Advanced Master Mix (Applied Biosystems $^{\mathrm{TM}}$ ) was used with TaqMan primers (Hs01060665_g1 for ACTB and Hs01377184_m1 for ASTILCS, both from ThermoFisher) (Fig. 2B). $\beta$-actin mRNA was used as a housekeeping control. The RNA levels were first normalized to the level of $\beta$-actin and then to an average value of the control group. All SYBR Green primers are listed in Supplemental Table 7.

\section{LNA transfection}

LNA gapmers targeting ASTILCS, SLC45A, PTK2, and PTP4A3 genes were custom-designed using Qiagen's Antisense LNA GapmeR designer (Supplemental Table 4), and non-targeting LNA gapmer (Negative Control A (NCA)) was included as a control. LNA gapmers were resuspended in water to a final concentration of $50 \mu \mathrm{M}$. 10,000 HUH7 cells were plated per well in a 96-well plate and incubated overnight. LNA gapmers were formulated with Lipofectamine 2000 (Invitrogen) in Opti-MEM $\left(\mathrm{Gibco}^{\mathrm{TM}}\right)$ according to the manufacturer. Each well was treated with $50 \mu \mathrm{l}$ Opti-MEM containing $20 \mathrm{pmol}$ LNA gapmer formulated and $0.25 \mu \mathrm{l}$ Lipofectamine 2000. Cell survival and gene expression were measured $24 \mathrm{~h}$ after transfection.

\section{Apoptosis analysis}

It was performed using In Situ Cell Death Detection Kit, TMR red (Roche) according to manufacturers' instructions. Briefly, cells were collected using $0.25 \%$ Trypsin-EDTA solution $\left(\mathrm{Gibco}^{\mathrm{TM}}\right)$, fixed with $4 \%$ paraformaldehyde (Electron Microscopy Sciences) in PBS $\left(\mathrm{Gibco}^{\mathrm{TM}}\right)$ at RT for $30 \mathrm{~min}$ and permeabilized with $0.1 \%$ Triton X-100 (Sigma) in 0.1\% sodium citrate (Sigma) for $2 \mathrm{~min}$ on ice. Next, TUNEL reaction mixture was added to the cells and incubated at $37^{\circ} \mathrm{C}$ for $60 \mathrm{~min}$. TMB-positive cells were detected and counted using BD FACSCelesta Flow Cytometer, at least 10,000 cells were analyzed per sample. 


\section{Cell cycle analysis}

It was performed using Click-iT EdU Alexa Fluor 647 Flow Cytometry Assay Kit (Invitrogen) and FxCycle Violet Stain (Invitrogen) according to manufacturers' instructions. Briefly, cells were labeled with $10 \mu \mathrm{M}$ EdU for $45 \mathrm{~min}$. Then the cells were harvested using a $0.25 \%$ Trypsin-EDTA solution $\left(\right.$ Gibco $\left.^{\mathrm{TM}}\right)$, fixed with Click-iT fixative at RT for $15 \mathrm{~min}$ protected from light and permeabilized with Click-iT saponin-based permeabilization and wash reagent. Next, click-iT ${ }^{\circledR}$ reaction cocktail was added to the cells and incubated for $30 \mathrm{~min}$ at RT protected from light. Finally, $1 \mu \mathrm{L}$ of FxCycle ${ }^{\mathrm{TM}}$ Violet stain was added to each sample and incubated for $30 \mathrm{~min}$ at RT protected from light. Fluorescence was analyzed using BD FACSCelesta Flow Cytometer, at least 10,000 cells were analyzed per sample.

\section{Statistical analysis}

Statistical significance was calculated using GraphPad Prism 8.2 package. The sample size was chosen based on preliminary results and similar publications. ROUT test $(Q=1 \%)$ was used to identify and remove outliers. D'Agostino-Pearson omnibus normality test was used to establish whether or not the population is distributed normally. Unpaired Mann-Whitney test was used to calculate the difference between two different populations which are not normally distributed. One-way analysis of variance (ANOVA) followed by Dunnett's post hoc test was used for multiple comparisons analysis of normally distributed populations with equal variances (i.e. equal standard deviations (SD)). Brown-Forsythe and Welch ANOVA tests followed by Dunnett's T3 multiple comparisons analysis were used for normally distributed populations with different SDs. Kruskal-Wallis test followed by Dunn's multiple comparisons analysis was used for populations which are not normally distributed.

\section{Results}

Pooled RNAi-based screen identifies IncRNAs potentially essential for HCC cell survival

To design the shRNA library, we performed transcriptome analysis in HUH7 HCC cell line and identified 1618 non-coding RNA transcripts longer than 200 base pairs and expressed at a level higher than 5 FPKM (Supplemental Fig. 1, Supplemental Table 1). Next, we constructed a library of 7873 shRNA vectors to knockdown the identified lncRNAs based on RNAi and applied on HUH7 cells (Fig. 1A). Each lncRNA was targeted by 4-5 shRNAs to account for shRNA off-target effects. To identify lncRNAs important for $\mathrm{HUH} 7$ cell survival, shRNAs present in the final population were compared to shRNA representation in the input library. A lncRNA was considered a candidate when at least two of its corresponding shRNAs were underrepresented in the final population with $\log _{2}$ (fold change compared to control) $\geq 1$ or by at least three shRNAs with $\log _{2}$ (fold change compared to control) $\geq 0.75$ (Fig. 1B). With these constraints, we identified seven IncRNA candidates for further validation (ENST00000429829, ENST00000510145, ENST00000457084, ENST00000501440.1, ENST0000036 6097.2, ENST00000518090, and ENST00000421703.5). To the best of our knowledge only ENST00000429829 and ENST00000510145 were previously characterized ${ }^{48-54}$.

Both of these IncRNAs have been identified in the context of cancer. Although their mechanisms are the focus of active discussion, their presence among our screen hits supports the likelihood that the rest of the transcripts are also involved in HCC cell survival and biology. ENST00000429829 is one of the multiple transcripts of gene ENSG00000229807, also known as XIST. In addition to its established role as the master regulator of X chromosome inactivation ${ }^{55}$, XIST has been reported to participate in progression of a variety of cancers, including $\mathrm{HCC}^{48-52}$. However, the results of these studies are controversial ${ }^{48-52}$. ENST00000510145 is one of nine transcripts of gene ENSG00000250682, also known as LINC00491 or BC008363. This gene was found to be upregulated in a TCGA colon adenocarcinoma dataset and was associated with lower patient survival, implying ENSG00000250682 importance for colorectal cancer progression $^{53}$. Conversely, in pancreatic ductal adenocarcinoma patients LINC00491 expression was significantly lower compared to the control group and was associated with better survival rates ${ }^{54}$.

\section{Validation of the screen results identifies IncRNA ASTILCS as a new regulator of $\mathrm{HCC}$ cell survival}

To validate the screening results, we individually expressed the five library shRNAs for each of the seven candidate IncRNAs (Supplemental Table 2) and repeated the screen in an arrayed format (Fig. 1C). Those lncRNAs for which at least two corresponding shRNAs reduced cell survival by more than $50 \%$ compared to the control shRNAs were selected for further analysis; these were ENST00000501440.1, ENST00000366097.2, ENST00000518090, and ENST00000421703.5 (Fig. 2A).

RNAi-based gene silencing is associated with a few pitfalls, particularly off-target activity and variability in knockdown efficiency ${ }^{56,57}$. We therefore further validated the candidate lncRNAs using an arrayed screen based on CRISPRi (Fig. 1C). To do so, we designed five sgRNAs (Supplemental Table 3) to allow targeting of each candidate lncRNA by CRISPRi (Fig. 2B, C). Among the four studied lncRNAs, CRISPRi-mediated knockdown of only ENST00000501440.1 and ENST00000366097.2 resulted in substantially decreased survival for HUH7 HCC cells (Fig. 2B, C). Specifically, 5/5 sgRNAs targeting lncRNA ENST00000501440.1 decreased HCC cell survival by 


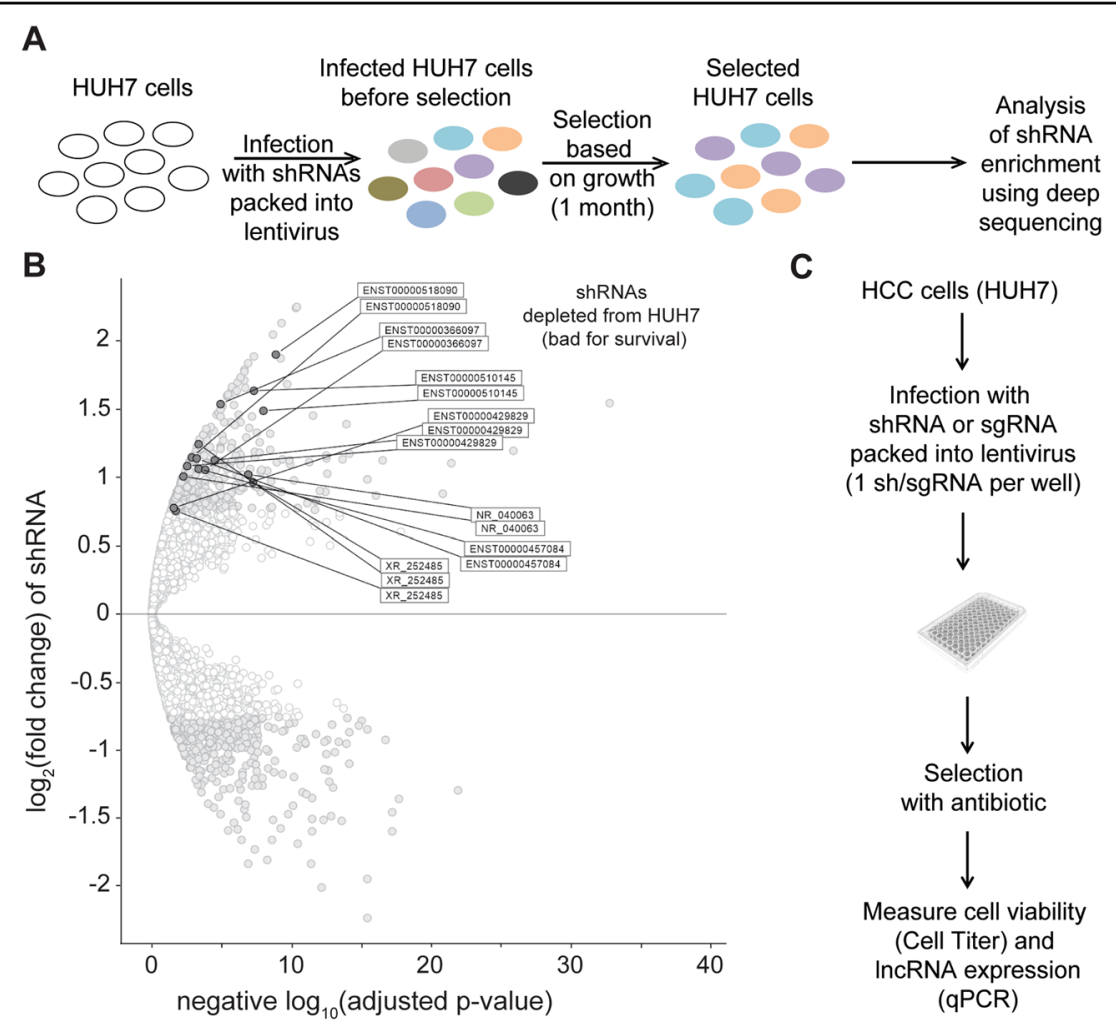

Fig. 1 Experimental design and selection strategy for the identification of IncRNAs essential for HUH7 HCC cell survival. A Schematic workflow of the survival-based pooled shRNA library screen in HUH7 cells. shRNAs were designed to target IncRNAs identified in the cell line. B Volcano plot of the differentially expressed shRNAs in the final population of HUH7 cells. The $x$-axis indicates the adjusted $p$-values plotted in $-\log _{10}$. The $y$-axis indicates the $\log _{2}$ (fold change) in shRNA expression, which was defined as the ratio of normalized shRNA expression in the input library over the final HUH7 population. Light gray dots represent shRNAs with $\log _{2}$ (fold change) $\geq 0.75$ and adjusted $p$-value $\leq 0.05$. Dark gray dots represent shRNAs of IncRNAs for which at least two shRNAs have $\log _{2}$ (fold change) $\geq 1$ and adjusted $p$-value $\leq 0.05$ or IncRNAs for which at least three shRNAs have $\log _{2}($ fold change $) \geq 0.75$ and adjusted $p$-value $\leq 0.05$. C Schematic workflow of arrayed shRNA and sgRNA screens used for validation of IncRNAs identified in $\mathbf{B}$.

more than $70 \%$ and $4 / 5$ sgRNAs targeting lncRNA ENST00000366097.2 resulted in more than 50\% HUH7 cell death. In contrast, knockdown of ENST00000518090 was not associated with a notable decrease in HCC cell survival and only $2 / 5$ sgRNAs designed to target ENST00000421703.5 induced partial lncRNA knockdown with mild effects on HCC cell survival. Discrepancy between the survival data obtained with RNAi and CRISPRi for ENST00000518090 and ENST00000421703 (Fig. 2A versus Fig. 2B) can be due to the differences in the mechanisms of the knockdown methods. In fact, multiple comparison studies have been published demonstrating that these approaches are complementary and not always identical, especially in case of lncRNAs ${ }^{21,58}$. Based on these results we concluded that ENST00000501440.1 and ENST00000366097.2 gene expression is critical for HCC cell survival. ENST00000501440.1 is the only transcript of ENSG00000244998 gene. It is a 1380 bp long antisense transcript comprised of two exons. ENST00000366097.2 is one of two transcripts of ENSG00000203266 gene. It is a $770 \mathrm{bp}$ long intergenic lncRNA consisting of three exons. Both transcripts (ENST00000501440.1 and ENST00000366097.2) are predicted to have low coding potential and are not conserved in chimpanzee or mouse $^{59}$. Thus, we identified two novel lncRNA genes which expression is potentially important for HCC cell survival.

To determine whether these two lncRNAs are HCC specific or are present in healthy liver tissues, we examined ENST00000501440.1 and ENST00000366097.2 gene expression in tissue samples from patients with HCC using a dataset from The Cancer Genome Atlas (TCGA-LIHC-rnaexp, downloaded from The Atlas of NcRNA in Cancer (TANRIC) $)^{60}$. We found that ENST00000501440.1 expression was significantly higher in liver cancer samples compared to the adjacent tissue (Fig. 2D; $p<0.0001$ ). These data suggest that only ENST00000501440.1 expression is critical for the survival of tumor cells. Yet, lncRNA expression was not associated 


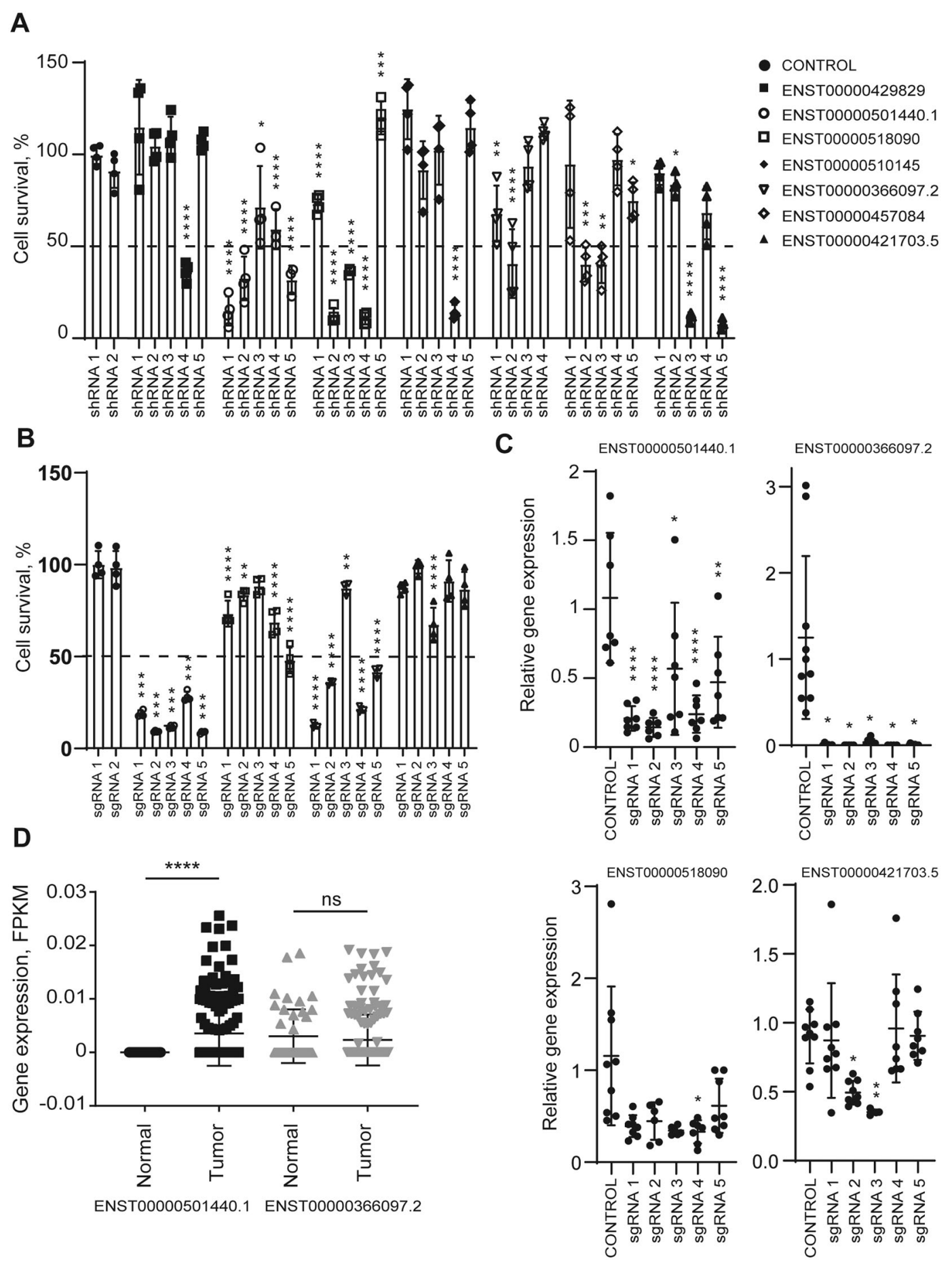

Fig. 2 Validation of the screen results identifies IncRNA ASTILCS a new regulator of HCC cell survival. A HUH7 cell survival upon shRNAmediated knockdown of candidate IncRNAs (compared to control shRNA1), $n \geq 3$. B HUH7 cell survival upon CRISPRi-mediated knockdown of IncRNA candidates from $\mathbf{A}$ compared to control sgRNA1, $n \geq 3$. C LncRNA expression in HUH7 cells transduced with sgRNA-dCas9-KRAB targeting one of the IncRNA candidates, $n \geq 4$. D ENST00000501440.1 (ASTILCS) and ENST00000366097.2 expression in HCC vs. adjacent tissue in patient samples (TCGALIHC-rnaexp downloaded from TANRIC), $n \geq 45$. All values are mean \pm SD, ${ }^{* * *}-p<0.0001 ;{ }^{* * *}-p<0.001 ;{ }^{* *}-p<0.01$; $^{*}-p<0.05$. 
with patient survival (Supplemental Fig. 2; long-rank $p$-value $=0.5)^{60}$. Because only ENST00000501440.1 expression is differentially expressed in cancer cells, we selected it for further analysis. Through the rest of the publication, we refer to this lncRNA by the name of ASTILCS (AntiSense Transcript Important for Liver Carcinoma Survival).

An insight into ASTILCS locus revealed that ASTILCS is an antisense sequence to the protein-coding gene Protein Tyrosine Phosphatase Type IVA 3 (PTP4A3) (Supplemental Fig. 3). PTP4A3 is important for cell proliferation; its knockdown decreases cell survival in multiple types of cells ${ }^{61-65}$. Because sgRNAs targeting ASTILCS bind PTP4A3 between 512 and 611 bp away from the transcription start site, there is a possibility that the sgRNA-dCas9-KRAB complex hinders PTP4A3 mRNA expression, resulting in HCC cell death independently of ASTILCS. Indeed, gene expression analysis of the sgRNA-treated cells revealed deep knockdown of PTP4A3 (Supplemental Fig. 4). To add orthogonal evidence of ASTILCS prosurvival effects on HCC cells, we knocked down its expression by transient transfection of antisense oligonucleotides containing locked nucleic acid modifications (LNA) (Supplemental Table 4). LNA gapmers bind with high affinity to complementary RNA sequences forming DNA.RNA hybrids, which are recognized and cleaved by RNAse H1, resulting in efficient gene knockdown ${ }^{66}$. We observed reduction in HUH7 HCC cell survival upon treatment with the LNA gapmers (Fig. 3A), which was associated with ASTILCS knockdown (Fig. 3B). We noticed that, despite a decrease in cell survival in LNA gapmer 2-treated samples, ASTILCS RNA levels in these samples were not significantly affected. These findings may be explained by previous reports demonstrating that antisense oligonucleotide hybridization with RNA can affect its function without inducing degradation ${ }^{67,68}$. Thus, LNA gapmer 2 binding to ASTILCS might perturb its function via steric blocking of lncRNA secondary structure formation or interaction with molecules important for the lncRNA signaling ${ }^{69,70}$. To further corroborate whether ASTILCS expression is critical for HCC cell survival, we measured its expression in HUH7 HCC cells transfected with the three most efficient shRNAs from the library (Supplemental Fig. 5) and observed dosage-dependent decrease in HCC cell survival (Figs. 3C and 2A). These findings substantiate that ASTILCS regulates HCC cell survival and its specific knockdown leads to HCC cell death independently of its reciprocal sense coding gene, PTP4A3.

\section{LncRNA ASTILCS knockdown in HCC cells results in apoptosis induction}

To understand the molecular mechanism of ASTILCS effects on HUH7 cell survival, we studied whether
ASTILCS knockdown affects HUH7 cell cycle distribution and apoptosis. To that end, we performed an EdUFxCycle Violet assay to determine ASTILCS effects on cell cycle. We did not observe any changes in cell cycle distribution which would correlate with ASTILCS knockdown efficacy in samples treated with shRNAs or with LNA gapmers compared to untreated control (Supplemental Fig. 6). Thus, we concluded that ASTILCS knockdown does not affect HUH7 cell proliferation in 24-48 h after transfection. Even if ASTILCS influence the transcription of cell cycle regulators, the effect can be postponed until a protein regulator will be degraded.

Next, we performed a TUNEL assay to assess ASTILCS knockdown effects on HUH7 cell apoptosis. We found that transformation with shRNA expressing plasmids or treatment with LNA gapmers led to a dose-dependent increase in the number of apoptotic cells (Fig. 3D and Supplemental Fig. 7). Differences in the apoptotic cell number between shRNA and LNA gapmer-treated samples were likely due to experimental constraints in the knockdown techniques. Apoptosis levels in LNA gapmertreated samples were measured $24 \mathrm{~h}$ after the treatment, while in shRNA-treated samples apoptosis could only be measured 4 days after transduction, providing time for compensation mechanisms to occur. Moreover, cell media in shRNA-treated samples had to be changed to remove the lentiviral particles and add selective agent, which could also result in partial removal of poorly attached apoptotic cells. From our findings we conclude that ASTILCS knockdown results in the induction of apoptosis and a subsequent decrease in HUH7 cell survival.

\section{ASTILCS is a nuclear antisense transcript which functions in} cis

As subcellular localization can hint towards the molecular mechanism of a lncRNA, we measured ASTILCS transcript levels in nuclear and cytoplasmic extracts and found ASTILCS RNA to be strongly enriched in the nucleus (Fig. 3E). These results are in line with the relatively low expression level of ASTILCS transcript in HUH7 cells ( 23.5 FPKM, Supplemental Table 5), a common feature of nuclear transcripts. Further, to classify the mechanism by which ASTILCS knockdown decreases HCC cell survival, we determined whether ASTILCS functions in cis or trans. To do so, we overexpressed cDNA encoding ASTILCS from a randomly integrated lentivirus and assessed cell proliferation as the population doubling time $\left(T_{\mathrm{d}}\right)$. We found that the $T_{\mathrm{d}}$ of cells overexpressing ASTILCS (1.13 \pm 0.07 days) was similar to the $T_{\mathrm{d}}$ of control cells expressing GFP from the same vector (1.13 \pm 0.03 days, $p=0.17$ ) (Fig. 3F,G and Supplemental Table 6). Because we did not observe any gain in survival for cells overexpressing ASTILCS, we concluded that 

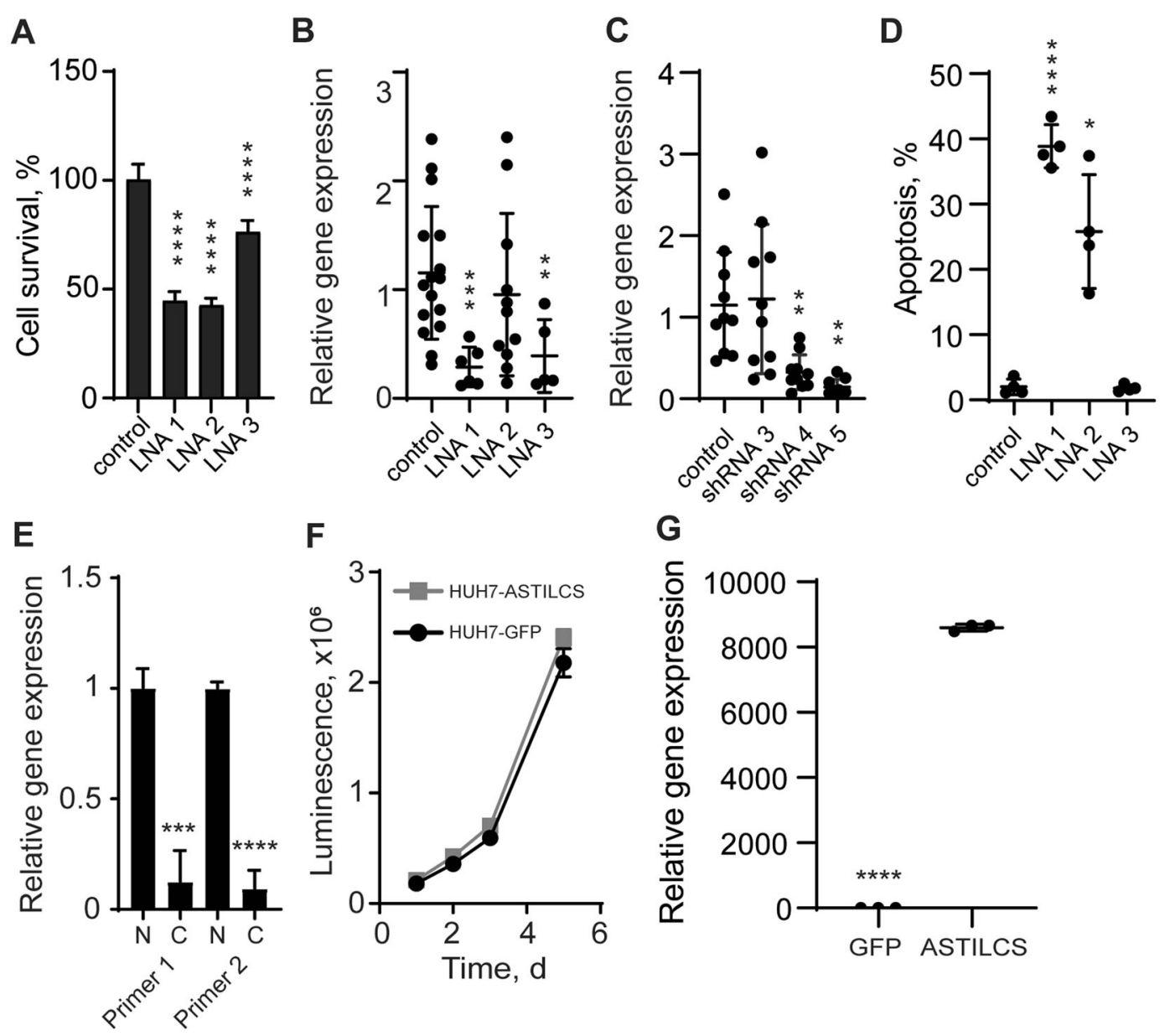

$\mathbf{F}$
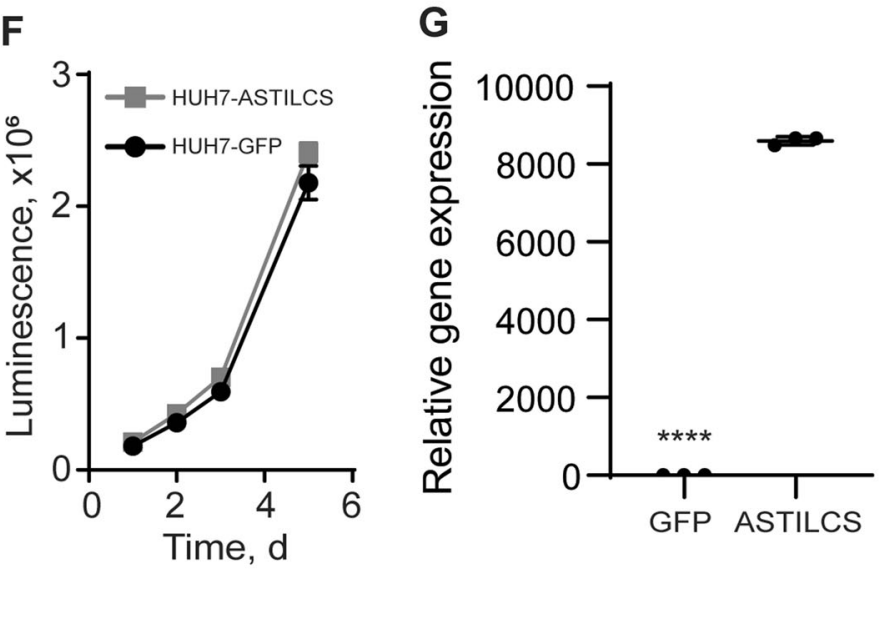

Fig. 3 ASTILCS expression is essential for liver carcinoma cell survival. A HUH7 cell survival $48 \mathrm{~h}$ after transfection with LNA gapmers targeting ASTILCS, $n \geq 6$. B ASTILCS expression in HUH7 cells transfected with LNA gapmers targeting ASTILCS, $n \geq 5$. C ASTICLS expression in HUH7 cells transduced with shRNAs targeting ASTILCS, $n \geq 5$. D Apoptosis in HUH7 cells treated with LNA gapmers targeting ASTILCS, $n=3$. E ASTILCS expression in HUH7 cell nucleus and cytoplasm, $n \geq 8$. F Growth curve for HUH7 cells transfected with GFP- or ASTILCS-expressing plasmids. G ASTILCS expression in HUH7 cells transduced with GFP- or ASTILCS-TRC209, $n=3$. All values are mean \pm SD, ${ }^{* * *}-p<0.0001 ; *^{* * *}-p<0.001 ;{ }^{*}-p<$ 0.01 ; $^{*}-p<0.05$; ns. $-p>0.05$.

ASTILCS is not likely to act in trans and that its effects on HCC cell survival are probably associated with cis functions.

\section{ASTILCS silencing is associated with downregulation of neighboring gene PTK2 essential for HCC cell survival}

The effects of low abundance nuclear cis-acting lncRNAs occur typically in the loci from which they are transcribed. Those effects can be mediated by: (1) the lncRNA transcripts themselves or in complexes with proteins like PRC2; (2) the act of lncRNA transcription; or (3) the regulatory DNA elements within the lncRNA locus $^{70,71}$. To determine whether the investigated phenotype might result from ASTILCS transcript effects on local gene expression, we examined the impact of ASTILCS knockdown on the expression of all genes within $1 \mathrm{Mb}$ of the target site (Fig. 4A). Analysis of the
HUH7 HCC cell transcriptome revealed that G proteincoupled receptor 20 (GPR20) and Maestro Heat Like Repeat Family Member 5 (MROH5) are not expressed in HUH7 cells (Supplemental Table 6), so they were removed from consideration. We found that LNA gapmer-induced ASTILCS knockdown led to a change in expression of all studied genes in the locus (Fig. 4B). Only downregulation of Solute Carrier Family 45 Member 4 (SLC45A4), Protein Tyrosine Kinase 2 (PTK2), DENN Domain Containing 3 (DENND3), and Trafficking Protein Particle Complex 9 (TRAPPC9) was associated with a dose-dependent decrease in both ASTILCS expression and HCC cell survival (Fig. 4B, see also Fig. 3A, B). In contrast, shRNA-mediated knockdown of ASTILCS was associated with gene expression downregulation of SLC45A, PTK2, and Chromatin accessibility complex protein 1 (CHRAC1) (Fig. 4C, see also Figs. 2A, 3C). 


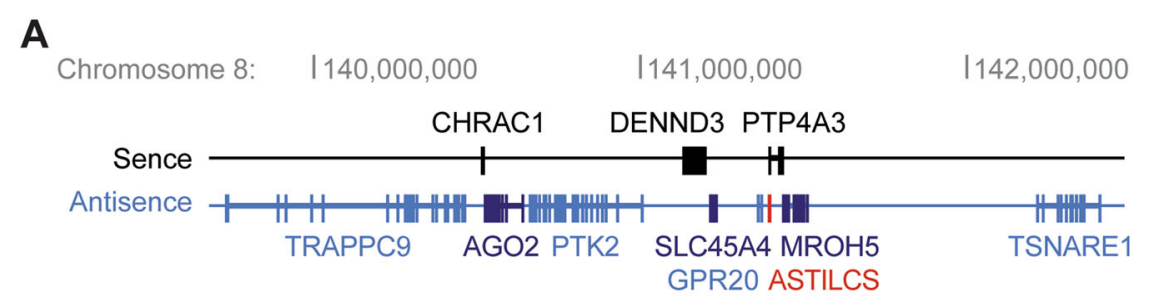

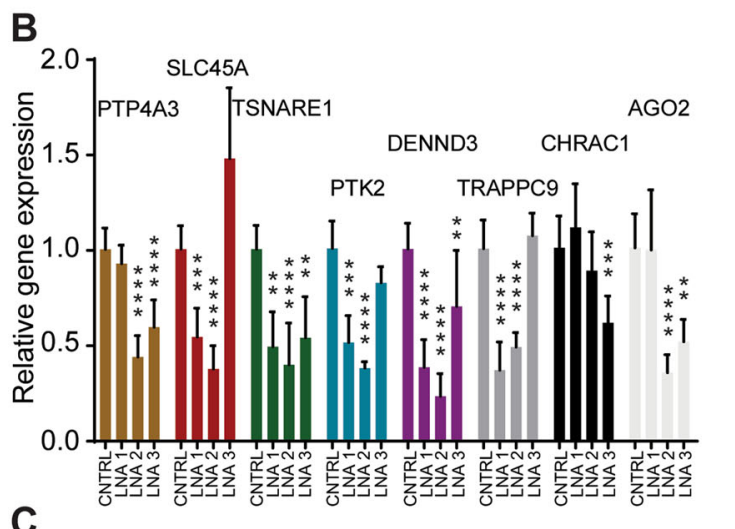
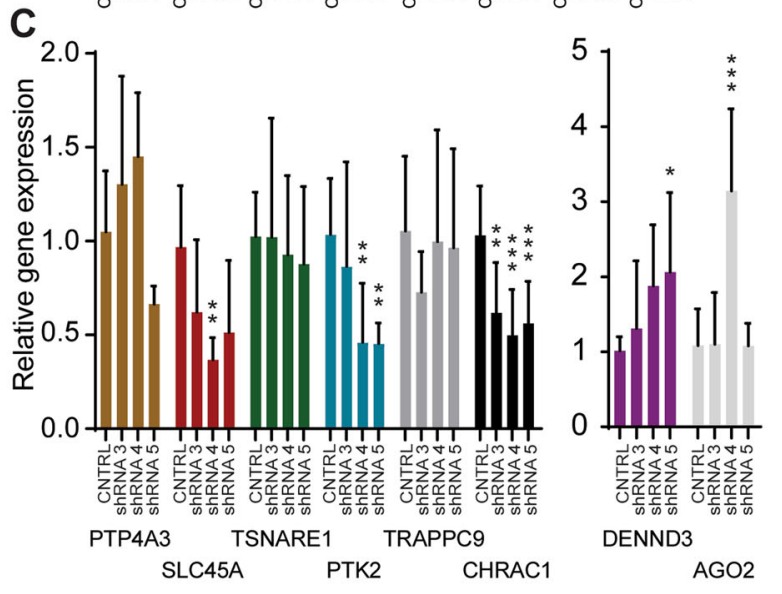

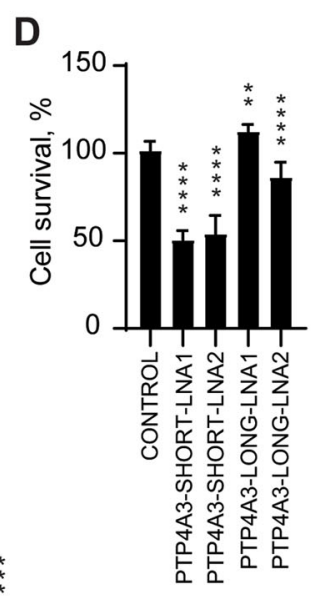

E
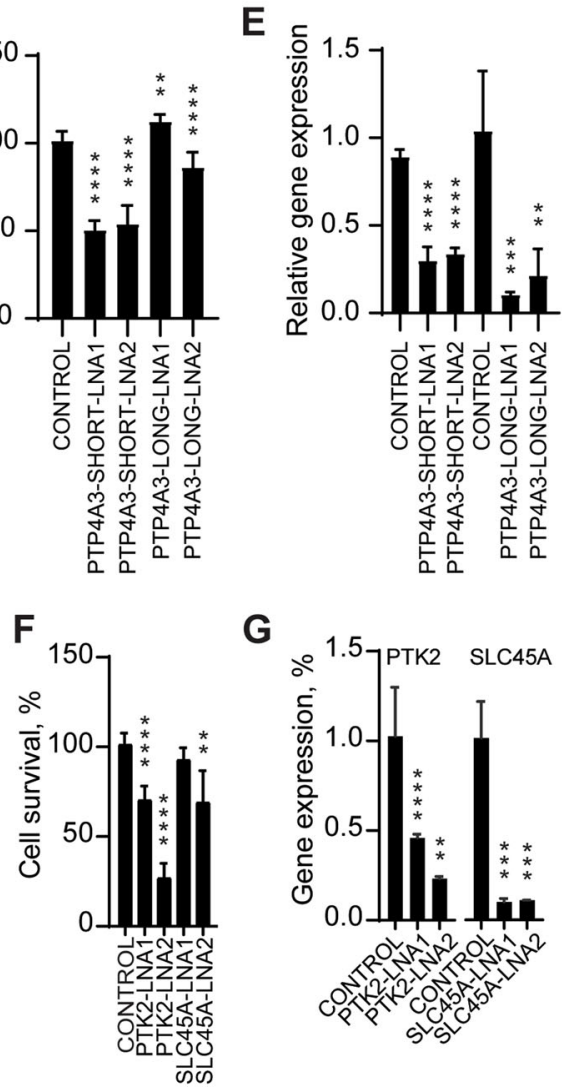

G

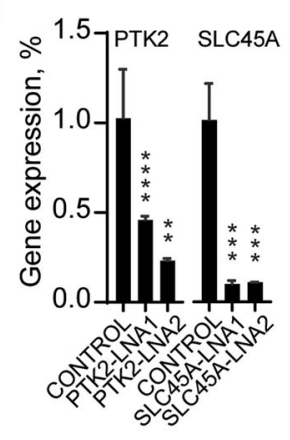

Fig. 4 Knockdown of ASTILCS results in dose-dependent downregulation of neighboring genes. A Genomic locus of ASTILCS. RNA expression of ASTILCS neighboring genes in HUH7 cells upon LNA gapmer-mediated $(n \geq 8)$ (B) or shRNA-mediated $(n \geq 5)$ (C) knockdown of ASTILCS. Cell survival $(n \geq 8)(\mathbf{D})$ and gene expression $(n \geq 5)(\mathbf{E})$ upon LNA-mediated silencing of PTP4A3 isoforms. Cell survival $(n \geq 9)(\mathbf{F})$ and gene expression $(n \geq$ 5) (G) upon LNA gapmer-mediated silencing of ASTILCS neighboring genes PTK2 or SCL45A, $n \geq 9$. All values are mean \pm SD, ${ }^{* * * *}-p<0.0001 ; *^{* *}-p$ $<0.001 ;{ }^{* *}-p<0.01 ;{ }^{*}-p<0.05$.

Expression of genes that was inconsistent across ASTILCS knockdown approaches was considered to result from indirect or off-target effects. Because only SLC45A and PTK2 mRNA expression was affected in the same manner by both shRNAs and LNA gapmers, we inferred that ASTILCS knockdown potentially induces HCC cell death by downregulating expression of one or both of these genes.

LncRNAs transcribed in opposite (antisense) direction to protein coding genes are often found to regulate activity of their sense pair in different manners ${ }^{72,73}$. Surprisingly, even though protein-coding gene PTP4A3 is located antisense to ASTILCS, we did not observe an apparent effect of ASTILCS knockdown on PTP4A3 mRNA expression (Fig. 4B, C). This fact indicates that the ASTILCS transcript itself does not affect the expression of PTP4A3 gene. Next, we studied whether PTP4A3 knockdown can affect HUH7 HCC cell survival. PTP4A3 produces six transcripts (T1-6), three longer (T3-5) than others (T1,2,6) (Supplemental Fig. 8); the sequence of only the long transcripts overlaps with ASTILCS. We designed LNA gapmers targeting long isoforms of PTP4A3 (T3-5) -PTP-LONG-LNA and LNA gapmers targeting two $(T 1,2)$ out of three short isoforms of PTP4A3. We could 
not design an LNA gapmer targeting only isoform T6 because it completely overlaps with the long isoforms. Interestingly, we found that knockdown of only the short PTP4A3 isoforms led to a dose-dependent decrease in HCC cell survival (Fig. 4D, E). Intrigued by the results, we also analyzed whether knockdown of the long PTP4A3 isoforms can affect the gene expression of the short isoforms. With this mechanism in mind, we measured the expression of the short isoforms in HUH7 HCC cells treated with LNA gapmers targeting long isoforms and observed no difference in the transcript expression (Supplemental Fig. 9). Because ASTILCS overlaps only with the long PTP4A3 isoforms and their knockdown does not affect the expression of their short, survival modulating counterparts, we conclude that ASTILCS silencing does not lead to a decrease in cell survival via downregulation of PTP4A3 transcripts.

Finally, we studied whether RNA knockdown of SLC45A and PTK2 itself can decrease HCC cell survival. PTK2 has been previously shown to affect HCC cell survival, with PTK2 silencing in HepG2 and HUH6 HCC cells lines reducing cell growth and inducing apoptosis ${ }^{74}$. Meanwhile, SLC45A4 has not been reported to affect cell survival. We treated HUH7 HCC cells with LNA gapmers targeting SLC45A or PTK2 and measured cell survival. We observed that only knockdown of PTK2 gene was associated with a decrease in HCC cell survival (Fig. 4F, G). Based on our results we conclude that ASTILCS knockdown might decrease HUH7 cell survival and induce apoptosis via downregulation of PTK2.

\section{Discussion}

Despite recent progress in HCC management, it remains the second deadliest cancer type with a 5-year relative patient survival rate of only $18 \%^{1,2}$. A better understanding of HCC biology informs the development of more efficient treatment strategies. An increasing number of studies suggests a vital role for lncRNAs in HCC progression ${ }^{75-77}$. However, their functions in HCC biology remain largely unexplored. To address that problem, in our study, we performed an shRNA-based pooled functional genetic screen to find lncRNAs that play crucial roles in HCC cell progression. Applying stringent filtering criteria and three-step validation we identified lncRNA ASTILCS (ENST00000501440.1) to be important for survival of HCC cells. To the best of our knowledge, we provide the first characterization of the lncRNA ASTILCS. Following a framework suggested by Joung et al. in ref. ${ }^{23}$ we determined that ASTILCS is a nuclear IncRNAs with a local regulatory mechanism. Using gene manipulation techniques, we demonstrated that ASTILCS loss-of-function results in apoptosis and downregulation of the neighboring gene PTK2, suggesting a possible mechanism of ASTILCS antisurvival effect.
PTK2, also known as Focal Adhesion Kinase, is a protein tyrosine kinase that plays an essential role in formation of cell-matrix junctions (focal adhesions), regulation of cell migration, and viability in a variety of cell types ${ }^{78}$. PTK2 recruitment to focal adhesions triggers PTK2 phosphorylation, creating a docking site for SH2 domain-containing proteins (Grb2, Shc, etc.), thus, linking PTK2 to the activation of the pro-proliferative and antiapoptotic RAS pathway ${ }^{78}$. Besides that, under certain cellular stress conditions, PTK2 can be recruited to the nucleus to facilitate Mdm2-dependent ubiquitination of tumor suppressor protein p53 and downregulate apoptosis $^{79}$. Multiple studies report on the importance of PTK2 for cancer progression ${ }^{80,81}$. To date a few PTK2 inhibitors have been studied in clinical trials, however, the best observed response was stable disease ${ }^{82-84}$. Understanding of mechanisms of PTK2 regulation might help to develop more effective PTK2-targeting therapies. Recently, two independent scientific groups simultaneously demonstrated that PTK2 is essential for HCC formation and growth in vivo because of its role in activation of the $W N T / \beta$-catenin signaling. PTK2 overexpression stimulated $\beta$-actin accumulation in the cell nucleus, thereby enhancing transcription of $\beta$-actin target genes and promoting hepatocarcinogenesis. PTK2 silencing, on the other hand, led to increase in apoptosis and a decrease in tumor growth ${ }^{85,86}$. Thus, downregulation of PTK2 mRNA expression by ASTICLS knockdown can be an important factor mediating the mechanism of ASTILCS' proapoptotic effect in HCC cells.

The molecular mechanisms of ASTILCS increasing PTK2 mRNA expression will require further studies. Epigenetic regulation might be one of the possible mechanisms. PTK2 is overexpressed in $30-60 \%$ of HCC patients and is associated with a higher metastasis rate and reduced survival. Meanwhile, PTK2 expression in healthy liver tissues is negligible, which underlines the importance of PTK2 expression for HCC progression ${ }^{87,88}$. In this study, we found that ASTILCS levels were also significantly increased in HCC samples compared to normal tissues. Interestingly, DNA sequence analysis in HCC patient samples revealed that PTK2 is amplified in $19-26 \%$ of cases, but mutated only in $2.5 \%{ }^{5,87}$. Therefore, there should be additional epigenetic mechanisms activating PTK2 expression. Examination of the PTK2 promoter demonstrated that the total methylation level of its CpG islands negatively correlated with PTK2 gene expression. Thus, promoter demethylation might be a mechanism of PTK2 overexpression. Indeed, treatment of HCC cells with a demethylation agent has shown to increase PTK2 mRNA and protein levels ${ }^{85}$. Some lncRNAs are known to affect DNA methylation via direct interaction with DNA methyltransferases (DNMTs) or via indirect recruitment of DNMTs through an intermediate 
protein $^{89}$. Hence, the aforementioned evidence creates a possibility that ASTILCS can increase PTK2 mRNA expression via regulation of its promoter methylation. More work is needed to explore molecular mechanism underling this process.

In addition to studies how ASTILCS effects on PTK2 RNA expression, we explored ASTILCS relationship with other neighboring genes. One of them, SLC45A4, is a proton-associated sucrose transporter, for which there are no reports of direct association with cancer or cell survival (PubMed search on 08-Jan-2021). In this study, we demonstrate for the first time, that ASTILCS knockdown leads to SLC45A4 gene silencing and that SLC45A4 silencing does not affect cell survival in HCC cells. Surprisingly, we did not observe a direct linkage between knockdown of antisense lncRNA ASTILCS and expression of its sense protein-coding pair, PTP4A3 gene. Thus, we inferred that the decrease in HCC cell survival upon ASTILCS knockdown is not likely mediated by changes in PTP4A3 mRNA expression. PTP4A3, also known as Phosphatase of Regenerating Liver 3 (PRL-3), is a protein-tyrosine phosphatase implicated in both cell proliferation and invasion in several types of cancer, including $\mathrm{HCC}^{64,65}$. Despite the importance of PTP4A3 for HCC cell survival, it seems the pro-survival effect of PTP4A3 is not regulated by ASTICLS RNA expression. Yet, this does not exclude the existence of other regulatory mechanisms between ASTILCS and PTP4A3, nor their importance in still undiscovered cell functions. Interestingly, the functional analysis of PTP4A3 transcripts presented here suggests that different transcripts affect cell survival in different ways in HCC cells. For the first time we report that only knockdown of short PTP4A3 transcripts (T1 and T2) reduces the cell survival, while expression of the long transcripts (T3-T5) has no effect on cell viability. This finding is in concordance with functional duality of PTP4A3, which is reported to regulate both cell survival and metastasis. Given only the expression of short transcripts correlates with cell survival, we can speculate that long transcripts might be involved in cell motility and invasion. This hypothesis requires further exploration.

In summary, we identified and characterized lncRNA ASTILCS, which regulates HCC cell survival presumably via activation of PTK2 mRNA expression and induction of apoptosis. In addition, we unveiled the effects of ASTILCS neighboring genes, PTK2, SLC45A4, and PTP4A3, on HCC cell survival. These findings provide valuable information about HCC biology and can advance the development of future HCC treatments.

\section{Acknowledgements}

We thank the Koch Institute Swanson Biotechnology Center for technical support, specifically bioinformatics \& computing, flow cytometry, genomics (BioMicro Center) and high throughput screening. We also thank Prof. Philip
Sharp (MIT) for valuable discussions of the project, and Dr. Elena Smekalova (MIT), Dr. Mikhail Nesterchuk (Skoltech) and Dr. Olga Sergeeva (Skoltech) for technical support at different stages of the project.

\section{Funding}

This work was supported by the MIT-Skoltech Initiative, Defense Advanced Research Projects Agency (W32P4Q-13-1-001), S. Leslie Misrock Frontier Research Fund for Cancer Nanotechnology, Translate Bio (Lexington, MA), and Koch Institute Support (core) Grant P30-CA14051 from National Cancer Institute.

\begin{abstract}
Author details
${ }^{1}$ David H. Koch Institute for Integrative Cancer Research, Massachusetts Institute of Technology, Cambridge, MA 02142, USA. ${ }^{2}$ Skolkovo Institute of Science and Technology, Moscow 121205, Russia. ${ }^{3}$ Department of Electrical Engineering and Computer Science, Massachusetts Institute of Technology, Cambridge, MA 02142, USA. ${ }^{4}$ Department of Biology, Massachusetts Institute of Technology, Cambridge, MA 02142, USA. Institute for Medical Engineering and Science, Massachusetts Institute of Technology, Cambridge, MA 02139, USA. ${ }^{6}$ Harvard and MIT Division of Health Science and Technology, Massachusetts Institute of Technology, Cambridge, MA 02139, USA.

${ }_{7}^{7}$ Department of Chemical Engineering, Massachusetts Institute of Technology, Cambridge, MA 02139, USA
\end{abstract}

\section{Author contributions}

V.K., D.G.A., R.B., and Y.R. designed the study; R.B. performed RNA sequencing, managed RNAi library design and edited the manuscript; Y.R. executed the rest of the experiments, analyzed data, and wrote the manuscript; J.T.G. assisted with the experiments and edited the manuscript; Y.L.D. assisted with the experiments; V.P.C. contributed to experimental design and execution and edited the manuscript; C.W. analyzed next-generation sequencing data and edited the manuscript; T.Z., V.K., and D.G.A. supervised the study and edited the manuscript.

\section{Data availability}

The sequence data has been submitted to the Gene Expression Omnibus under superseries identifier GSE152651 which consists of the RNA-Seq data (GSE152650) and the shRNA screen data (GSE152649). Original data and numbers for tables are uploaded to Mendeley Data (https://doi.org/10.17632/ dggchs5s8m.1).

\section{Conflict of interest}

The authors declare that they have no conflict of interest.

Ethics statement

Not applicable.

\section{Publisher's note}

Springer Nature remains neutral with regard to jurisdictional claims in published maps and institutional affiliations.

Supplementary information The online version contains supplementary material available at https://doi.org/10.1038/s41419-021-03453-w.

Received: 15 July 2020 Revised: 6 January 2021 Accepted: 12 January 2021 Published online: 15 February 2021

\section{References}

1. Allemani, C. et al. Global surveillance of trends in cancer survival 2000-14 (CONCORD-3): analysis of individual records for 37?513?025 patients diagnosed with one of 18 cancers from 322 population-based registries in 71 countries. Lancet 391, 1023-1075 (2018).

2. Villanueva, A. Hepatocellular carcinoma. N. Engl. J. Med. 380, 1450-1462 (2019).

3. Rawla, P., Sunkara, T., Muralidharan, P. \& Raj, J. P. Update in global trends and aetiology of hepatocellular carcinoma. Wspclczesna Onkol. 22, 141-150 (2018). 
4. Allain, C., Angenard, G., Clément, B. \& Coulouarn, C. Integrative genomic analysis identifies the core transcriptional hallmarks of human hepatocellular carcinoma. Cancer Res. 76, 6374-6381 (2016).

5. Kan, Z. et al. Whole-genome sequencing identifies recurrent mutations in hepatocellular carcinoma. Genome Res. 23, 1422-1433 (2013).

6. Nakagawa, H., Fujita, M. \& Fujimoto, A. Genome sequencing analysis of liver cancer for precision medicine. Semin. Cancer Biol. 55, 120-127 (2019).

7. Schulze, K., Nault, J. C. \& Villanueva, A. Genetic profiling of hepatocellular carcinoma using next-generation sequencing. J. Hepatol. 65, 1031-1042 (2016).

8. Okrah, K. et al. Transcriptomic analysis of hepatocellular carcinoma reveals molecular features of disease progression and tumor immune biology. $n p j$ Precis. Oncol 2, 25 (2018).

9. Harding, J. J. et al. Prospective genotyping of hepatocellular carcinoma: clinical implications of next-generation sequencing for matching patients to targeted and immune therapies. Clin. Cancer Res. 25, 2116-2126 (2019).

10. The ENCODE Project Consortium. An integrated encyclopedia of DNA elements in the human genome. Nature 489, 57-74 (2012).

11. Derrien, T. et al. The GENCODE v7 catalog of human long noncoding RNAs: analysis of their gene structure, evolution, and expression. Genome Res. 22 1775-1789 (2012).

12. Iyer, M. K. et al. The landscape of long noncoding RNAs in the human transcriptome. Nat. Genet. 47, 199-208 (2015).

13. Yang, Y. et al. Recurrently deregulated IncRNAs in hepatocellular carcinoma. Nat. Commun. 8, 14421 (2017).

14. Cui, H. et al. A comprehensive genome-wide analysis of long noncoding RNA expression profile in hepatocellular carcinoma. Cancer Med. 6, 2932-2941 (2017).

15. Wong, C.-M., Tsang, F. H.-C. \& Ng, I. O.-L. Non-coding RNAs in hepatocellular carcinoma: molecular functions and pathological implications. Nat. Rev. Gastroenterol. Hepatol. 15, 137-151 (2018).

16. McDonel, P. \& Guttman, M. Approaches for understanding the mechanisms of long noncoding RNA regulation of gene expression. Cold Spring Harb. Perspect. Biol. 11, a032151 (2019).

17. Carlevaro-Fita, J. \& Johnson, R. Global positioning system: understanding long noncoding RNAs through subcellular localization. Mol. Cell 73, 869-883 (2019).

18. Engreitz, J. M. et al. Local regulation of gene expression by IncRNA promoters, transcription and splicing. Nature 539, 452-455 (2016).

19. Noh, J. H., Kim, K. M., McClusky, W. G., Abdelmohsen, K. \& Gorospe, M. Cytoplasmic functions of long noncoding RNAs. Wiley Interdiscip. Rev. RNA 9, e1471 (2018).

20. Root, D. E., Hacohen, N., Hahn, W. C., Lander, E. S. \& Sabatini, D. M. Genomescale loss-of-function screening with a lentiviral RNAi library. Nat. Methods $\mathbf{3}$ 715-719 (2006).

21. Gilbert, L. A. et al. Genome-scale CRISPR-mediated control of gene repression and activation. Cell 159, 647-661 (2014)

22. Beermann, J. et al. A large shRNA652 library approach identifies IncRNA Ntep as an essential regulator of cell proliferation. Cell Death Differ. 25 307-318 (2018)

23. Joung, J. et al. Genome-scale activation screen identifies a IncRNA locus regulating a gene neighbourhood. Nature 548, 343-346 (2017).

24. Tiessen, I. et al. A high-throughput screen identifies the long non-coding RNA DRAIC as a regulator of autophagy. Oncogene 38, 5127-5141 (2019).

25. Cai, P. et al. A genome-wide long noncoding RNA CRISPRi screen identifies PRANCR as a novel regulator of epidermal homeostasis. Genome Res. 30, 22-34 (2020).

26. Galeev, R. et al. Genome-wide RNAi screen identifies cohesin genes as modifiers of renewal and differentiation in human HSCs. Cell Rep. 14, 2988-3000 (2016).

27. Kampmann, M. et al. Next-generation libraries for robust RNA interferencebased genome-wide screens. Proc. Natl Acad. Sci. USA 112, E3384-E3391 (2015).

28. Castel, S. E. \& Martienssen, R. A. RNA interference in the nucleus: roles for small RNAs in transcription, epigenetics and beyond. Nat. Rev. Genet. 14, 100-112 (2013).

29. Avivi, S. et al. Visualizing nuclear RNAi activity in single living human cells. Proc. Natl. Acad. Sci. USA 114, E8837-E8846 (2017).

30. Lennox, K. A. \& Behlke, M. A. A direct comparison of anti-microRNA oligonucleotide potency. Pharm Res. 2, 1788-1799 (2010).

31. Goyal, A. et al. Challenges of CRISPR/Cas9 applications for long non-coding RNA genes. Nucleic Acids Res. 45, e12 (2017)

32. Langmead, B., Trapnell, C., Pop, M. \& Salzberg, S. L. Ultrafast and memoryefficient alignment of short DNA sequences to the human genome. Genome Biol. 10, R25 (2009).
33. Li, B. \& Dewey, C. N. RSEM: accurate transcript quantification from RNA-Seq data with or without a reference genome. BMC Bioinform. 12, 323 (2011).

34. Li, C. et al. Long non-coding RNA XIST promotes TGF- $\beta$-induced epithelial-mesenchymal transition by regulating miR-367/141-ZEB2 axis in non-small-cell lung cancer. Cancer Lett. 418, 185-195 (2018).

35. Xing, F. et al. Loss of XIST in breast cancer activates MSN-c-Met and reprograms microglia via exosomal miRNA to promote brain metastasis. Cancer Res. 78, 4316-4330 (2018)

36. Moffat, J. et al. A lentiviral RNAi Library for human and mouse genes applied to an arrayed viral high-content screen. Cell 124, 1283-1298 (2006).

37. Marcel, M. Cutadapt removes adapter sequences from high-throughput sequencing reads. EMBnet. journal, [S.I.], v. 17, n. 1, p. pp. 10-12, may 2011. ISSN 2226-6089.

38. Li, H. \& Durbin, R. Fast and accurate short read alignment with Burrows-Wheeler transform. Bioinformatics 25, 1754-1760 (2009).

39. $\mathrm{Li}, \mathrm{H}$. et al. The Sequence Alignment/Map format and SAMtools. Bioinformatics 25, 2078-2079 (2009).

40. Love, M. I., Huber, W. \& Anders, S. Moderated estimation of fold change and dispersion for RNA-seq data with DESeq2. Genome Biol. 15, 550 (2014).

41. Wiederschain, D. et al. Single-vector inducible lentiviral RNAi system for oncology target validation. Cell Cycle 8, 498-504 (2009).

42. Doench, J. G. et al. Optimized sgRNA design to maximize activity and minimize off-target effects of CRISPR-Cas9. Nat. Biotechnol. 34, 184-191 (2016).

43. Sanson, K. R. et al. Optimized libraries for CRISPR-Cas9 genetic screens with multiple modalities. Nat. Commun. 9, 5416 (2018).

44. Thakore, P. I. et al. Highly specific epigenome editing by CRISPR-Cas9 repressors for silencing of distal regulatory elements. Nat. Methods $\mathbf{1 2}$, 1143-1149 (2015).

45. Ran, F. A. et al. Genome engineering using the CRISPR-Cas9 system. Nat. Protoc. 8, 2281-2308 (2013).

46. Stewart, S. A. et al. Lentivirus-delivered stable gene silencing by RNAi in primary cells. RNA 9, 493-501 (2003).

47. Sancak, Y. et al. The Rag GTPases bind raptor and mediate amino acid signaling to mTORC1. Science 320, 1496-1501 (2008).

48. Lin, X. Q., Huang, Z. M., Chen, X., Wu, F. \& Wu, W. XIST induced by JPX suppresses hepatocellular carcinoma by sponging miR-155-5p. Yonsei Med. J. 59, 816-826 (2018).

49. Kong, Q. et al. LncRNA XIST functions as a molecular sponge of miR-194-5p to regulate MAPK1 expression in hepatocellular carcinoma cell. J. Cell. Biochem. 119, 4458-4468 (2018).

50. Chang, S., Chen, B., Wang, X., Wu, K. \& Sun, Y. Long non-coding RNA XIST regulates $\mathrm{PTEN}$ expression by sponging miR-181a and promotes hepatocellular carcinoma progression. BMC Cancer 17, 248 (2017).

51. Ma, W. et al. Downregulation of long noncoding RNAs JPX and XIST is associated with the prognosis of hepatocellular carcinoma.Clin. Res. Hepatol. Gastroenterol. 41, 163-170 (2017).

52. Zhuang, L. K. et al. MicroRNA-92b promotes hepatocellular carcinoma progression by targeting Smad7 and is mediated by long non-coding RNA XIST. Cell Death Dis. 7, e2203-e2203 (2016).

53. Wan, J. et al. LINC00491 as a new molecular marker can promote the proliferation, migration and invasion of colon adenocarcinoma cells. Onco Targets Ther. 12, 6471-6480 (2019).

54. Li, J. et al. Long non-coding RNAs expressed in pancreatic ductal adenocarcinoma and IncRNA BC008363 an independent prognostic factor in PDAC. Pancreatology 14, 385-390 (2014).

55. Sahakyan, A., Yang, Y. \& Plath, K. The role of Xist in X-chromosome dosage compensation. Trends Cell Biol. 28, 999-1013 (2018).

56. Chen, D. et al. Long noncoding RNA XIST expedites metastasis and modulates epithelial-mesenchymal transition in colorectal cancer. Cell Death Dis. 8, e3011-e3011 (2017).

57. Kaelin, W. G. Jr. Use and abuse of RNAi to study mammalian gene function: molecular biology. Science 337, 421 (2012).

58. Stojic, L. et al. Specificity of RNAi, LNA and CRISPRi as loss-of-function methods in transcriptional analysis. Nucleic Acids Res. 46, 5950-5966 (2018).

59. Volders, P.-J. et al. LNCipedia 5: towards a reference set of human long noncoding RNAs. Nucleic Acids Res. 47, D135-D139 (2019).

60. Li, J. et al. TANRIC: an interactive open platform to explore the function of InCRNAs in cancer. Cancer Res. 75, 3728-3737 (2015).

61. Lian, Y.-X., Chen, R., Xu, Y.-H., Peng, C.-L. \& Hu, H.-C. Effect of protein-tyrosine phosphatase $4 \mathrm{~A} 3$ by small interfering RNA on the proliferation of lung cancer. Gene 511, 169-176 (2012). 
62. Zhang, J. et al. miR-21, miR-17 and miR-19a induced by phosphatase of regenerating liver-3 promote the proliferation and metastasis of colon cancer. Br. J. Cancer 107, 352-359 (2012).

63. Matsukawa, Y. et al. Constitutive suppression of PRL-3 inhibits invasion and proliferation of gastric cancer cell in vitro and in vivo. Pathobiology 77, 155-162 (2010)

64. Zhao, W.-B., Li, Y., Liu, X., Zhang, L.-Y. \& Wang, X. Evaluation of PRL-3 expression, and its correlation with angiogenesis and invasion in hepatocellular carcinoma. Int. J. Mol. Med. 22, 187-192 (2008).

65. Li, B.-H. et al. Up-regulation of phosphatase in regenerating liver-3 (PRL-3) contributes to malignant progression of hepatocellular carcinoma by activating phosphatase and tensin homolog deleted on chromosome Ten (PTEN)/phosphoinositide 3-kinase (PI3K)/AKT signaling path. Med. Sci. Monit. 24, 8105-8114 (2018).

66. Crooke, S. T. et al. Molecular mechanisms of antisense oligonucleotides. Nucleic Acid Ther. 27, 70-77 (2017).

67. Mologni, L., leCoutre, P. \& Nielsen, P. E. G.P. C. Additive antisense effects of different PNAs on the in vitro translation of the PML/RARalpha gene. Nucleic Acids Res. 26, 1934-1938 (1998).

68. Baker, B. F. et al. 2'-O-(2-methoxy)ethyl-modified anti-intercellular adhesion molecule 1 (ICAM-1) oligonucleotides selectively increase the ICAM-1 mRNA level and inhibit formation of the ICAM-1 translation initiation complex in human umbilical vein endothelial cell. J. Biol. Chem. 272, 11994-2000 (1997).

69. Bennett, C. F. \& Swayze, E. E. RNA targeting therapeutics: molecular mechanisms of antisense oligonucleotides as a therapeutic platform. Annu. Rev. Pharmacol. Toxicol. 50, 259-263 (2010).

70. Gil, N. \& Ulitsky, I. Regulation of gene expression by cis-acting long non-coding RNAs. Nat. Rev. Genet. 21, 102-117 (2020).

71. Kopp, F. \& Mendell, J. T. Functional classification and experimental dissection of long noncoding RNAs. Cell 172, 393-407 (2018).

72. Khorkova, O., Myers, A. J., Hsiao, J. \& Wahlestedt, C. Natural antisense transcripts. Hum. Mol. Genet. 23, R54-R63 (2014).

73. Pelechano, V. \& Steinmetz, L. M. Gene regulation by antisense transcription. Nat. Rev. Genet. 14, 880-893 (2013).

74. Gnani, D. et al. Focal adhesion kinase depletion reduces human hepatocellular carcinoma growth by repressing enhancer of zeste homolog 2. Cell Death Differ. 24, 889-902 (2017).
75. Klingenberg, M., Matsuda, A., Diederichs, S. \& Patel, T. Non-coding RNA in hepatocellular carcinoma: mechanisms, biomarkers and therapeutic targets. J. Hepatol. 67, 603-618 (2017).

76. Lanzafame, M., Bianco, G., Terracciano, L. M., Ng, C. K. Y. \& Piscuoglio, S. The role of long non-coding RNAs in hepatocarcinogenesis. Int. J. Mol. Sci. 19, 682 (2018).

77. Li, C. et al. Progress and prospects of long noncoding RNAs (IncRNAs) in hepatocellular carcinoma. Cell. Physiol. Biochem. 36, 423-434 (2015).

78. Schaller, M. D. Cellular functions of FAK kinases: insight into molecular mechanisms and novel functions. J. Cell Sci. 123, 1007-1013 (2010).

79. Lim, S.-T. et al. Nuclear FAK promotes cell proliferation and survival through FERM-enhanced p53 degradation. Mol. Cell 29, 9-22 (2008).

80. Sulzmaier, F. J., Jean, C. \& Schlaepfer, D. D. FAK in cancer: mechanistic findings and clinical applications. Nat. Rev. Cancer 14, 598-610 (2014).

81. Panera, N., Crudele, A., Romito, I., Gnani, D. \& Alisi, A. Focal adhesion kinase: insight into molecular roles and functions in hepatocellular carcinoma. Int. J. Mol. Sci. 18, 99 (2017).

82. Shimizu, T. et al. A first-in-Asian phase 1 study to evaluate safety, pharmacokinetics and clinical activity of VS-6063, a focal adhesion kinase (FAK) inhibitor in Japanese patients with advanced solid tumors. Cancer Chemother. Pharmacol. 77, 997-1003 (2016).

83. Brown, N. F. et al. A study of the focal adhesion kinase inhibitor GSK2256098 in patients with recurrent glioblastoma with evaluation of tumor penetration of [11C]GSK2256098. Neuro Oncol. 20, 1634-1642 (2018).

84. Jones, S. F. et al. A phase I study of VS-6063, a second-generation focal adhesion kinase inhibitor, in patients with advanced solid tumors. Investig. New Drugs 33, 1100-1107 (2015).

85. Fan, Z. et al. PTK2 promotes cancer stem cell traits in hepatocellular carcinoma by activating Wnt/ $\beta$-catenin signaling. Cancer Lett. 450, 132-143 (2019).

86. Shang, $N$. et al. Focal adhesion kinase and $\beta$-catenin cooperate to induce hepatocellular carcinoma. Hepatology 70, 1631-1645 (2019).

87. Fujii, T. et al. Focal adhesion kinase is overexpressed in hepatocellular carcinoma and can be served as an independent prognostic factor. J. Hepatol. 41, 104-111 (2004).

88. Itoh, S. et al. Role of expression of focal adhesion kinase in progression of hepatocellular carcinoma. Clin. Cancer Res. 10, 2812-2817 (2004).

89. Zhao, Y., Sun, H. \& Wang, H. Long noncoding RNAs in DNA methylation: new players stepping into the old game. Cell Biosci. 6, 45 (2016). 